\title{
A INTEGRAÇÃO ENSINO-SERVIÇO- COMUNIDADE NO CURSO DE ENFERMAGEM: O QUE DIZEM OS ENFERMEIROS PRECEPTORES
}

Elaine Cristina Dias Franco

Vânia Aparecida da Costa Oliveira ${ }^{1}$

Briene Leandro Lopes ${ }^{1}$

Victoria da Cunha Avelar ${ }^{2}$ http://orcid.org/0000-0001-8744-7726

http://orcid.org/0000-0001-7082-5997

http://orcid.org/0000-0002-6935-2202

http://orcid.org/0000-0002-7987-7651

Objetivo: analisar a percepção do enfermeiro preceptor de campo de estágio acerca da integração ensino-serviço-comunidade no curso de Enfermagem de uma universidade pública do estado de Minas Gerais. Metodologia: Estudo qualitativo desenvolvido, no período de dezembro de 2017 a março de 2018, por meio de entrevistas com 12 enfermeiros preceptores. A análise dos dados ocorreu por meio da Análise de Conteúdo. Resultados: A integração ensino-serviço favorece a articulação teórico-prática, o ensino contextualizado e a aproximação com a realidade dos serviços de saúde. Há desafios relacionados com os serviços de saúde como a falta de planejamento e a indisponibilidade de tempo e; outros relacionados à universidade como o distanciamento dos serviços de saúde, a descontinuidade das ações e a falta de capacitação dos preceptores. Considerações Finais: A integração ensino-serviço coaduna para a formação de profissionais que atendam as demandas do Sistema Único de Saúde e da sociedade atual.

Descritores: Enfermagem, Ensino Superior, Preceptoria.

\section{THE TEACHING-SERVICE-COMMUNITY INTEGRATION IN THE NURSING COURSE: WHAT THE PRECEPTING NURSES SAY}

Objective: To o analyze the perception of the preceptor nurse in the internship field about the teaching-service-community integration in the Nursing course of a public university in the State of Minas Gerais. Methodology: Qualitative study developed from Diciembre de 2017 a marzo de 2018, through interviews with 12 nurses preceptors. Data analysis occurred through Content Analysis. Results: The teaching-service integration favors the theoretical-practical articulation, the contextualized teaching and the approximation with the reality of health services. There are challenges related to health services such as lack of planning and unavailability of time and; others related to the university, such as the distancing of health services, the discontinuity of actions and the lack of training of preceptors. Final Considerations: The teaching-service integration is suitable for the formation of professionals that meet the demands of the Unified Health System and the current society.

Descriptors: Nursing, Education Higher, Preceptorship.

\section{LA INTEGRACIÓN ENSEÑANZA-SERVICIO-COMUNIDAD EN EL CURSO DE ENFERMERİA: LO QUE DICEN LAS ENFERMERAS PRECEPTORAS}

Objetivo: analizar la percepción del preceptor de enfermería del campo de pasantías sobre la integración enseñanza-servicio-comunidad en el curso de Enfermería de una universidad pública en el estado de Minas Gerais. Metodología: Estudio cualitativo desarrollado de febrero a mayo de 2018, a través de entrevistas con 12 enfermeros preceptores. El análisis de datos se realizó a través del análisis de contenido. Resultados: La integración del servicio-enseñanza favorece la articulación teórico- práctica, la enseñanza contextualizada y la aproximación a la realidad de los servicios de salud. Existen desafíos relacionados con los servicios de salud, como la falta de planificación y la falta de tiempo y; otros relacionados con la universidad, como el distanciamiento de los servicios de salud, la discontinuidad de las acciones y la falta de capacitación de los preceptores. Consideraciones finales: La integración del servicio-enseñanza es adecuada para la formación de profesionales que satisfagan las demandas del Sistema Único de Salud y la sociedad actual. Descritores: Enfermería, Educación Superior, Preceptoría.

IUniversidade Federal de São João Del Rei, MG, Brasil.

${ }^{2}$ Universidad Juárez del Estado de Durango (UJED), México.

Autor Correspondente: Elaine Cristina Dias Franco Email: elainefrancol@yahoo.com.br Recebido 29/1/20 Aceito: 22/4/20 


\section{INTRODUÇÃO}

Ao ser instituído, o Sistema Único de Saúde (SUS) apresenta dentre seus diversos desafios o alinhamento da formação profissional na área da saúde às necessidades da população e dos serviços de saúdel. Tal alinhamento é primordial, uma vez que os princípios e as diretrizes defendidas pelo SUS, como a humanização do cuidado e da assistência à saúde e o desenvolvimento de um trabalho ético, interprofissional e interdisciplinar em todos os pontos da Rede de Atenção à Saúde (RAS) precisam ser experienciados pelos estudantes durante todo o processo formativo, pois estes serão os futuros profissionais que irão atuar nos diversos serviços que compõem essa rede ${ }^{2}$

Diante disso, diversas iniciativas para a reestruturação de formação de recursos humanos para a área de saúde têm sido propostas ${ }^{3}$, dentre elas, destaca-se a integração ensino, serviço e comunidade, que pressupõe o desenvolvimento de um trabalho coletivo, pautado na pactuação e integração entre docentes e discentes dos cursos de formação na área da saúde, trabalhadores, gestores e usuários dos serviços, primando pela qualidade da atenção à saúde e a formação profissional de estudantes e trabalhadores dos serviços ${ }^{4-5}$.

Dessa forma, a integração ensino, serviço e comunidade mostra-se favorável à formação de profissionais de saúde no e para o SUS ao considerar que o cotidiano dos serviços de saúde são cenários singulares de formação․․, capaz de provocar mudanças nas práticas cotidianas dos profissionais de saúde, pois incita reflexões sobre a produção do cuidado e dessa forma, corrobora para a superação de ações meramente prescritivas já cristalizadas pelo modelo médico centrado 6 .

A partir dessa premissa, a formação em saúde pautada nessa integração tem sido discutida e sustentada pelas Diretrizes Curriculares Nacionais (DCN) dos cursos da área da saúde, a exemplo da Enfermagem ${ }^{7}$ e Medicina que trazem, em suas diretrizes, a valorização da inserção dos estudantes nos serviços de saúde, já nos primeiros períodos dos cursos. Parte-se do entendimento que a aproximação entre o ensino e o serviço favorece a vivência e a proposição de ações alicerçadas na promoção, proteção e reabilitação da saúde associada a ações que visem a prevenção de doenças e agravos ${ }^{8-9}$

Assim, reconhecendo-se a necessidade de os serviços públicos de saúde e as instituições de ensino superior (IES) compartilharem as responsabilidades pela formação e pela produção do cuidado², e, ainda, considerando-se que a ordenação de recursos humanos na área da saúde é uma atribuição do SUS, prevista na constituição Federal de 1988, os Ministérios da Saúde e da Educação desenvolveram estratégias para a condução dos processos de formação e qualificação profissional na saúde, dentre elas citam-se a Política de
Educação Permanente em Saúde (PNEPS) e o Programa Nacional de Reorientação da Formação Profissional em Saúde (PróSaúde), que possuem como pano de fundo a integração ensino, serviço e comunidade ${ }^{1}$.

Todavia, em que pesem os esforços empreendidos e os avanços alcançados a partir da implantação dessas políticas e das discussões, favoráveis a elas, realizadas no âmbito da formação superior em saúde, a integração ensino, serviço e comunidade ainda não é uma realidade que permeia os serviços de saúde e as IES.

Alguns estudos ${ }^{10-11-12}$ constataram que há ausência de uma parceria efetiva que contemple os interesses das IES e dos serviços de saúde. Dessa forma, não há uma contrapartida das IES para os serviços, sobressaindo, na maioria das vezes, os interesses das primeiras. Nesse caso, o sucesso do processo está comprometido, uma vez que a integração pressupõe conexão e relação entre as partes. Além disso, a resistência e ou indisponibilidade de alguns profissionais dos serviços de saúde, e a ausência de comunicação entre docentes e preceptores são algumas das dificuldades que têm impossibilitado a concretização da integração ensino, serviço e comunidade ${ }^{13}$

Diante desse cenário torna-se importante investigar os modos como os profissionais de saúde que atuam como preceptores, percebem a integração ensino-serviço nos seus cotidianos, considerando-se que eles são corresponsáveis pelo processo de ensino-aprendizagem e possuem papel fundamental na promoção da articulação teórico-prática. Dessa maneira, o presente estudo teve como objetivo analisar a percepção do enfermeiro, preceptor de campo de estágio, acerca da integração ensino-serviço-comunidade no curso de Enfermagem de uma Universidade Pública do estado de Minas Gerais.

\section{MÉTODO}

\section{Tipo de estudo}

Estudo descritivo com abordagem qualitativa que permite desvelar, na perspectiva de enfermeiros preceptores, os significados e intencionalidades que permeiam o cotidiano dos serviços de saúde quando estes são espaços de integração do ensino e serviço.

\section{Participantes}

Os participantes do estudo foram 12 enfermeiros preceptores de unidades de Atenção Primária a Saúde de um munícipio do estado de Minas Gerais. A seleção intencional dos participantes seguiu aos seguintes critérios: (I) Ser Enfermeiro com atuação como preceptor no curso de Enfermagem nos últimos doze meses; (II) Não estar afastado por qualquer motivo à época da coleta. 


\section{Cenário de Estudo}

O cenário de estudo foi o Curso de Graduação em Enfermagem de uma Instituição Federal (IF), localizada na região Centro Oeste do estado de Minas Gerais. O curso está organizado em nove períodos, nos quais os estudantes desde o primeiro período são inseridos em unidades de Atenção Primária à Saúde para vivenciar a articulação teórico-prática dos conteúdos apreendidos ao longo do curso

\section{Coleta de dados}

A coleta de dados ocorreu no período de dezembro de 2017 a março de 2018, por meio de entrevistas individuais realizadas nas unidades de saúde, em dia e horário previamente agendado. Após a assinatura do Termo de Consentimento Livre e Esclarecido (TCLE), as entrevistas foram gravadas e posteriormente transcritas na íntegra pelas pesquisadoras. Os depoimentos foram identificados com a letra $\mathrm{E}$ seguida do número de sequência das entrevistas (El, E2) garantindo a preservação do anonimato dos participantes.

\section{Análise dos dados}

Os dados foram analisados por meio da análise de conteúdo de Bardin, composta por 3 etapas: pré-análise; exploração do material e; tratamento dos dados e interpretação ${ }^{14}$. Emergiram dos dados empíricos duas categorias que foram analisadas a luz da literatura científica sobre a temática.

\section{Aspectos éticos}

Este estudo obteve aprovação do Comitê de Ética em Pesquisa com CAEE: 62368116.5.0000.5545 e parecer de aprovação $\mathrm{n}$ ㅇ 1.878 .176 emitido em 20 de dezembro de 2016 . $\mathrm{O}$ estudo atendeu a todas as prerrogativas da Resolução $466 / 2012$

\section{RESULTADOS}

A partir da análise qualitativa dos dados, os resultados se expressam nas categorias "Integração ensino-serviço: mundos entrelaçados" e "Integração ensino-serviço: desafios para a sua concretização" que são apresentadas a seguir:

\section{Integração ensino-serviço: mundos entrelaçados}

Em seus discursos os enfermeiros preceptores narram as suas percepções sobre a integração ensino-serviço e as relacionam com a superação da dualidade entre teoria e prática e consideram que o serviço é, para o estudante, um espaço favorável à aprendizagem e ao desenvolvimento crítico:

O aluno quando aproxima do serviço de saúde consegue ver a aplicação do conteúdo teórico ....fica mais próximo teoria e prática (El).

Quando está na unidade de saúde ele consegue integrar... né..., essa teoria a partir do que acontece no serviço, do cotidiano, dos desafios e também das conquistas diárias (E5).
Quando o aluno tem o contato desde o início, né, então na hora que ele chega lá na teoria que ele vai pensar, eu vi dessa forma na realidade, mas a teoria tá colocando dessa forma' então a possibilidade dele ter uma visão crítica em relação ao que está vendo na teoria, é muito maior, ele consegue dar sentido para a prática e teoria, né, o serviço torna-se o lugar de aprender também (El2).

Para os preceptores, o contato do estudante com a realidade dos serviços de saúde permite a ele contextualizar o que foi apreendido na sala de aula, conhecer os aspectos positivos, as dificuldades e os problemas existentes nesses serviços, bem como, os seus usuários e as atribuições cotidianas dos profissionais:

No serviço o aluno consegue ver a realidade, a teoria da sala de aula passa a ter sentido, ele passa a viver e entender o SUS, ele conhece o usuário e o que os profissionais fazem no seu dia-a-dia e até consegue ter um olhar crítico sobre o que está acontecendo nos serviços públicos hoje (Ell).

Ele vive o SUS, suas potências e suas mazelas ...eu acho que o aluno quando está aqui desde o inicio do curso, ele tem a oportunidade de desenvolver um olhar crítico sobre as politicas públicas, seus limites e também suas vantagens (E9).

Para outros preceptores, a presença do estudante promove troca de experiências e aprendizado mútuo. Além disso, a presença dele propicia uma assistência à saúde mais comprometida com as reais necessidades da população, estimula discussões e planejamentos das ações desenvolvidas pela equipe, bem como aproxima os profissionais de conhecimentos atualizados em suas áreas de atuação:

Eu acho que é uma troca né... o aluno vem pra ver a rotina, aprender com a experiência da gente, com o conhecimento e a gente aprende com os conhecimentos novos que ele vai trazendo né... eu acho que é isso a interação é essa (E6).

Eu recebo alunos aqui a mais ou menos seis anos e a presença deles provoca mudanças no serviço, ficamos mais atentos às necessidades da população, eles favorecem ações de promoção da saúde, participam de nossas reuniões e planejamentos semanais...acho que a equipe de forma geral fica mais atualizada e pros alunos também é bom porque eles aproximam da prática profissional de forma efetiva (E8).

Os alunos provocam mudanças na rotina dos 
serviços, nas práticas dos profissionais, eles nos aproximam de conhecimentos atualizados (E7)

\section{Integração ensino-serviço: desafios para a sua concre- tização}

Para os enfermeiros preceptores a concretização da integração ensino-serviço traz à tona diversos desafios para os serviços de saúde e para a universidade. No âmbito dos serviços de saúde há menção à necessidade de aprimoramento constante do planejamento das ações individuais e coletivas, ao envolvimento dos profissionais em atividades de ensino e à adequação de estrutura física para atender às necessidades didático-pedagógicas em determinados momentos:

Pra mim é um desafio a PIESC e o estágio...todo semestre eu ajusto minha agenda e o planejamento da unidade, deixo mais tempo entre consultas para que eu possa dedicar um tempo maior para os alu$\operatorname{nos}(E 12)$.

[...] às vezes mudo até o lugar de realizar as atividades para favorecer o professor e alunos (E3)

Por outro lado, no que diz respeito à universidade, os desafios apontados relacionam-se ao alinhamento da proposta pedagógica com a realidade dos serviços de saúde, a efetiva aproximação da instituição de ensino com os preceptores, a capacitação dos preceptores para atender às demandas da universidade, além da proposição e realização de intervenções nos serviços e comunidade de forma efetiva e continuada:

Como preceptora preciso ter conhecimento $e$ entender como será feito esse estágio e fazer um planejamento pra que isso ocorra de forma a ser uma via de mão dupla, com benefícios para o aluno e para a unidade, então é importante serviço e universidade ficar bem alinhado (E5).

Eu como preceptor, eu percebo a Federal muito distante da gente... eles deveriam ser mais próximos...muitas vezes o contato fica restrito ao período das aulas (ElO).

[...] eu acho seria importante ter momentos de capacitação dos profissionais que estão na rede, que são preceptores, para falarmos a mesma linguagem da universidade (Ell).

[...] termina o semestre eles vão embora e as vezes sem nem dar um retorno sobre as atividades desenvolvidas, quais intervenções foram feitas, como avaliam as ações e como a equipe pode dar continuidade (E2).

\section{DISCUSSÃO}

Verifica-se, neste estudo, que a inserção dos estudantes em unidades básicas de saúde já na etapa inicial do curso de Enfermagem é uma potente estratégia para o processo de ensino- aprendizagem, além disso, mostra-se favorável ao desenvolvimento das habilidades e competências inerentes ao profissional enfermeiro e à formação crítica-reflexiva, e assim, encontra-se alinhada às proposições das $\mathrm{DCN}^{15}$

Diversos estudos ${ }^{6,16-17}$ apontam os benefícios que uma parceria efetiva, entre as IES e os serviços de saúde, traz para o processo formativo, especialmente quando a corresponsabilização por esse processo encontra-se fortalecida.

Ao serem inseridos nos serviços de saúde, os estudantes aproximam-se do mundo do trabalho e de suas interfaces com os elementos que compõem o cotidiano da assistência à saúde e do cuidado, o que permite uma visão mais ampliada sobre as necessidades de saúde da população, das RAS e do SUS ${ }^{13,16}$, assegurando uma trajetória acadêmica diferenciada, e assim, favorecendo uma maior confiança para a futura atuação profissional nesses cenários ${ }^{18-19}$. Além disso, há que se considerar que a presença do estudante nos serviços de saúde promove rupturas na acomodação do fazer cotidiano de muitos profissionais, transformando, assim, a forma de pensar e agir sobre o cuidado em saúde ${ }^{11,15}$

Contudo, os dados encontrados nesse estudo põem em relevo a necessidade das IES e dos seus respectivos docentes refletirem e ressignificarem os seus papéis no fortalecimento da integração ensino, serviço e comunidade. De forma semelhante, o estudo de Marin e colaboradores ${ }^{20}$ mostrou que o alcance de uma integração efetiva depende de mudanças estruturais e funcionais significativas, da construção conjunta entre gestores, trabalhadores, docentes e estudantes, com franca aproximação de sujeitos do mundo do trabalho e do ensino, dois universos que nem sempre dialogam suficientemente para que ocorra a troca e a complementaridade dos saberes.

A falta de capacitações que contemplem o preparo didático-pedagógico do preceptor e o conhecimento da proposta pedagógica da IES é sem dúvida um fator limitante para o bom desempenho da preceptoria e melhor aproveitamento do estudante. Nesse sentido, torna-se imprescindivel que as IES não considerem somente as competências técnicas e a experiência profissional dos preceptores e assumam o compromisso de instrumentalizá-los para a formação de outros profissionais $^{21,4}$

A percepção, por parte da IES, dos docentes e estudantes, de que os serviços de saúde são apenas campos de estágio $^{16}$ precisa ser veementemente combatida. Em vez disso, tais serviços precisam ser valorizados e compreendidos 
como instâncias sem as quais tona-se impossivel aos estudantes adquirirem o preparo necessário para o pleno exercício da profissão. Para tanto, a presença da IES nos serviços de saúde e vice-versa, deve ser contínua, estendendo-se para além dos períodos de aulas. Nesse sentido, o trabalho conjunto pode ser garantido por meio do planejamento de atividades que englobem capacitações, seminários, discussões sobre Projetos Pedagógicos de Cursos (PPC), dentre outras.

Outro dado encontrado neste estudo e que merece destaque é o fato da IES não realizar, ao final do semestre e juntamente com os profissionais dos serviços de saúde, uma avaliação cuidadosa sobre as atividades e intervenções que foram realizadas. Em função disso, os profissionais ficam sem um direcionamento sobre o que deve ou não ter continuidade. Resultado semelhante foi encontrado no estudo ${ }^{16}$, realizado com enfermeiros docentes e enfermeiros assistenciais, onde os autores afirmam que nem sempre há retorno, pelos docentes e alunos, para a equipe, sobre o alcance dos objetivos do ensino no final do semestre.

Destaca-se, ainda, que o planejamento sistematizado, precedendo o início das atividades de ensino e a imersão dos estudantes nos serviços de saúde, pode favorecer a elaboração de uma agenda de atividades que atenda às necessidades dos serviços e seja, ao mesmo tempo, condizente com os conteúdos que estão sendo vistos pelos estudantes, e, por conseguinte, com as competências a serem adquiridas por eles, naquele momento.

Frente ao exposto, verifica-se, neste estudo, que os discursos produzidos pelos enfermeiros preceptores dos serviços de saúde ratificam a relevância que a integração, ensino e comunidade têm para a formação em saúde, por isso, ela deve ser compreendida como um ideal a ser perseguido por todos os que estejam envolvidos e comprometidos, direta ou indiretamente, com a qualidade da assistência à saúde, com a produção do cuidado e com a consolidação do SUS.

\section{Limitações do estudo}

Embora o estudo demonstre resultados significativos, considera-se como fator limitante o fato de ter sido desenvolvido apenas com os enfermeiros preceptores da Atenção Primária à Saúde, não contemplando outros cenários como as unidades hospitalares.

\section{Contribuições para a prática}

Os resultados dessa pesquisa corroboram com os achados de outros estudos da área, e, dessa forma, apontam a inexistência de uma parceria efetiva entre as instituições de ensino e os serviços de saúde. A principal contribuição do estudo é reiterar que a produção de mudanças nas práticas assistenciais de saúde, em consonância com as proposições do SUS, somente será alcançada se as instituições de ensino e de saúde assumirem o desafio de pensar e refletir conjuntamente sobre os problemas e as dificuldades encontradas, cotidianamente, nos cenários de ensino e de assistência à saúde, bem como, nas possibilidades e estratégias para enfrentamento.

Assim, se ambas as instituições encontram-se comprometidas com a vida humana, a ampliação dos canais de comunicação e o fortalecimento do diálogo e do trabalho coletivo, é condição sine qua non para dirimir o descompasso entre as proposições e demandas apresentadas por cada uma delas.

\section{CONSIDERAÇÕES FINAIS}

$O$ presente estudo permitiu inferir que a integração, ensino, serviço e comunidade apesar da reconhecida importância e relevância para a formação de profissionais de saúde capacitados para atuarem em consonância com os princípios do SUS apresenta fragilidades em diversas dimensões, como, por exemplo, a ausência de um diálogo constante entre IES e serviços de saúde, docentes e preceptores, a falta de capacitações didático-pedagógicas destes últimos, e a descontinuidade da presença da IES nos serviços de saúde, após o término do semestre.

A partir dos discursos dos enfermeiros preceptores verifica-se que a presença do estudante nos serviços de saúde desencadeia reflexões importantes nos profissionais, que passam a exercer suas atividades de forma mais atenciosa e adequada às necessidades da população. Por outro lado, para o estudante, estar imerso no cotidiano desses serviços constitui-se numa possibilidade singular de acionar diversos conhecimentos que foram apreendidos ao longo da sua formação e a contextualizá-los à realidade encontrada.

Dessa maneira, conclui-se que a integração ensino, serviço e comunidade, quando devidamente planejada possibilita ganhos a todos os envolvidos, sejam IES, docentes, estudantes, profissionais e comunidade. Portanto, de forma coletiva, todos devem empreender esforços para que ela, com toda a sua potencialidade, se concretize.

Contribuições dos Autores: ECDF: Concepção e desenho do estudo, análise e interpretação dos dados, redação, revisão crítica e revisão final do artigo. VACO: Coleta, análise e interpretação dos dados. BLL: Redação do artigo. VCA: Redação do artigo. 


\section{REFERÊNCIAS}

1 Vendruscolo C, Ferraz F, Prado ML do, Kleba ME, Reibnitz KS. Integração ensino-serviço e sua interface no contexto da reorientação da formação na saúde. Interface [Internet]. 2016[cited 2019 Jan 15]:20(59):1015-1025. Available from: http://www.scielo.br/pdf/icse/ v20n59/1807-5762-icse-1807-576220150768.pdf

2 Codato LAB, Garanhani ML, González AD, Carcereri DL, Carvalho BG, Morita MC. Significados do estágio em Unidades Básicas de Saúde para estudantes de graduação. Revista da ABENO [Internet]. 2019[cited 2019 Ago 22];19(1):2-9. Available from: https://revabeno. emnuvens.com.br/revabeno/article/view/662/517

3 Franco ECD, Soares AN, Gazzinelli MF. Macro and micropolitics recontextualization of an integrated curriculum: experienced itinerary in nursing undergraduate. Esc. Anna Nery [Internet].2018 [cited 2020 Jan 23];22(4):e20180053. Available from: http://www.scielo.br/pdf/ ean/v22n4/pt_1414-8145-ean-22-04-e20180053.pdf

4 Ministério da Saúde (BR). Portaria GM/MS no 1.996/07, de 20 de agosto de 2007. Dispõe sobre as diretrizes para a implementação da Politica Nacional de Educação Permanente em Saúde. Brasilia.[Internet] 2007 [cited 2020 Jan 23]. Available from: http://bvsms.saude. gov.br/bvs/saudelegis/gm/2007/prt1996_20_08_2007.html

5 Moraes JT, Silva AE, Gontijo TL, Ribeiro RF, Faria R das GS. Avaliação do impacto da capacitação no trabalho para o cuidado de pessoas com estomias. Enferm Foco [internet]. 2019 [cited 2020 Abril 01]; 10 (3): 93-98. Available from: http://revista.cofen.gov.br/index.php/ enfermagem/article/view/1810/575

6 Vasconcelos ACF de, Stedefeldt E, Frutuoso MFP. Uma experiência de integração ensino-serviço e a mudança de práticas profissionais: com a palavra, os profissionais de saúde. Interface [Internet]. 2016 [cited 2019 Jan 23];20(56): 147-158. Available from: http://www. scielo.br/pdf/icse/v20n56/1807-5762-icse-20-56-0147.pdf

7 Brasil. Resolução CNE/CES no1133 de 2001. Diretrizes Curriculares Nacionais dos Cursos de Graduação em Enfermagem. Diário Oficial da União, Brasília, 2001. Seção 1E, p. 131. ; [citado 2018 Dec 19]. Available from: http://portal.mec.gov.br/cne/arquivos/pdf/CESO3.pdf

8 Franco ECD, Soares AN, Bethony MFG. Curriculo integrado no ensino superior em enfermagem: o que dizem os enfermeiros docentes. Enferm Foco [Internet]. 2016 [cited 2019 Fev 22]; 7(1):33-36. Available from: http://revista.cofen.gov.br/index.php/enfermagem/ article/view/662/281.

9 Vieira LM, Sgavioli C de APP, Simionato EMRS, Inoue ESY, Heubel MTCD, Conti MHS de et al. Formação profissional e integração com a rede básica de saúde. Trab. educ. saúde [Internet]. 2016 [cited 2019 Dec 13];14(1):293-304. Available from: http://www.scielo.br/pdf/ tes/v14nl/1981-7746-tes-14-01-0293.pdf

10 Oliveira VA da C. Currículo do curso de enfermagem da Universidade Federal de Minas Gerais (EEUFMG) [manuscrito]: perspectiva de docentes e discentes. 2016. 125 f. Orientadora: Prof Dra Maria Flávia Gazzinelli Bethony. Tese (Doutorado). Universidade Federal de Minas Gerais, Escola de Enfermagem.

11 Brehmer LC de F, Ramos FRS. Teaching-service integration: implications and roles in experiences of Undergraduate Courses in Nursing. Rev. esc. enferm. USP [Internet]. 2014 [cited 2020 jan 24]; 48 (1): 118-124. Available from: http://www.scielo.br/pdf/reeusp/v48nl/ pt_0080-6234-reeusp-48-01-118.pdf

12 Dantas L dos S, Pereira RVS, Bernadino I de M, Figueiredo RCPP, Madruga RCR, Lucas RS de CC. Perfil de competências de preceptores para a Atenção Primária em Saúde. Revista da ABENO [internet]. 2019 [cited 2020 jan 24]:19(2):156-166. Available from: https:// revabeno.emnuvens.com.br/revabeno/article/view/677/590

13 Mendes T de MC, Bezerra H de S, Carvalho Y de M, Silva LG da, Souza CMC de L, Andrade FB de. Interação Ensino-Serviço-Comunidade no Brasil e o que dizem os atores dos cenários de prática: uma revisão integrativa. Revista Ciência Plural [Internet] 2018 [cited 2020 jan 24]:4(1) :98-116. Available from: https://periodicos.ufrn.br/rcp/article/view/14283

14 Bardin L. Análise de Conteúdo. Lisboa: Edições 70; 2016.

15 Peres CRFB, Marin MJS, Soriano ECI, Ferreira M de L da SM. A dialectical view of curriculum changes in nursing training. Rev. esc. enferm. USP [Internet]. 2018 [cited 2020 jan 24];52: e03397. Available from: http://www.scielo.br/pdf/reeusp/v52/1980-220X-reeusp-52-e03397.pdf

16 Andrade SR de Boehs AEB, CGE.Percepções de enfermeiros docentes e assistenciais sobre a parceria ensino-serviço em unidades básicas de saúde. Interface - Comunicação, Saúde, Educação [Internet]. 2015 [Cited 24 Jan 2020]; 19(54)537-547. Available from: http:// www.scielo.br/pdf/icse/v19n54/1807-5762-icse-19-54-0537.pdf

17 Albiero JFG, Freitas SFT de. Modelo para avaliação da integração ensino-serviço em Unidades Docentes Assistenciais na Atenção Básica. Saúde em Debate [Internet]. 2017 [cited 24 Jan 2020]; 41(114):753-767. Available from: http://www.scielo.br/pdf/sdeb/v4lnll4/ 0103-1104-sdeb-41-114-0753.pdf

18 Forte FDS, Pessoa TRRF, Freitas CHSM, Pereira CAL, Carvalho Junior PM. Reorienting dental education: the preceptor's view of supervised internship in the Brazilian Health System (SUS). Interface [Internet]. 2015 [cited 10 Jan 2020];19(Supl 1):831-43. Available from: http://www.scielo.br/pdf/icse/v19s1/1807-5762-icse-19-s1-0831.pdf

19 Souza LB , Bonamigo AW. Integração ensino-serviço na formação de profissionais para sistemas públicos de saúde. Trab. educ. saúde [Internet]. 2019 [cited 10 Jan 2020]; 17(3):e0021747. Available from http://www.scielo.br/pdf/tes/v17n3/1678-1007-tes-17-03-e0021747.pdf 20 Marin MJS, Oliveira MA de C, Otani MAP, Cardoso CP, Moravcik MYAD, Conterno L de $O$ et al. A integração ensino-serviço na formação de enfermeiros e médicos: a experiência da FAMEMA. Ciênc. saúde coletiva [Internet]. 2014 [cited 2020 Jan 24]; 19(3):967-974. Available from: http://www.scielo.br/pdf/csc/v19n3/1413-8123-csc-19-03-00967.pdf

21Valente GSC, Ferreira FC. Nurse knowledge and understanding for teaching and management in primary health care: a descriptive/ exploratory study. Online braz j nurs [Internet]. 2013 [cited 2020 Jan 24]; 12(Suppl): 653-55. Available from: http://www.objnursing.uff. br/index.php/nursing/article/view/4389/pdf_2 